\title{
Inhibitory Effect of Active Substances of Lollyfish (Holothuria atra) Against the Development of Plasmodium falciparum Based on In Silico Study
}

\author{
Felly Moelyadi*, Prawesty Diah Utami, Irmawati M. Dikman \\ Faculty of Medicine Hang Tuah University \\ Komplek Barat RSAL Dr. Ramelan Jl. Gadung No. 1, Jagir, Wonokromo, Surabaya, Jawa Timur, 60111 Indonesia \\ Email: moelyadifelly1@gmail.com
}

\begin{abstract}
The high level of artemisinin resistance as the antimalarial drug makes the active substances found of lollyfish (Holothuria atra) become a very useful discovery as a new antimalarial drug. The purpose of this research is to find out the inhibitory effect of the active substances of lollyfish against the development of Plasmodium falciparum with in silico method. This is a one-shot experimental study research. Based on the test of potentially active substances of Iollyfish through PubChem (https://pubchem.ncbi.nlm.nih.gov/), there are pyrogallol and catechin that have potential as the antimalarial drug. Pyrogallol, chlorogenic acid, catechin dan ascorbic acid have indirect inhibition to P. falciparum Orotidine 5-Monophosphate Decarboxylase (PfOMPDC) through carbon dioxide $\left(\mathrm{CO}_{2}\right)$ and it is visualized by STITCH DB Version 5.0 (http://stitch.embl.de/). The binding affinity score of catechin, obtained from molecular docking, is higher than other substances and artemisinin. The Physicochemical and pharmacokinetic activity of the substance was predicted through SWISS ADME (http://www.swissadme.ch/index.php), while the toxicity was predicted through Pro-Tox (http://tox.charite.de/protox_ll/). Catechin is a substance in lollyfish that is the safest because its lowest toxicity and very effective to be used as the antimalarial drug because of its high lethal dose 50 (LD50). Therefore, active substances in lollyfish have inhibitory effects against the development of P. falciparum based on in silico study.
\end{abstract}

Keywords: in silico, Iollyfish, malaria, PfOMPDC

\section{Introduction}

Malaria disease causes health problems in the world. Malaria is caused by a Plasmodium parasite that can live and develop in human red blood cells and transmitted by female Anopheles mosquito's bite. The high mortality rate of infant, toddler, and pregnant women and low productivity level of infected person are some of the problems caused by malaria (Putra, 2011). In 2018, there was 228.000 .000 cases of malaria disease that was reported by all countries with 405.000 global deaths. The biggest case of malaria was reported from Africa with 213.000 .000 cases or 93\% from all cases in the world and followed by Southeast Asia with $3.4 \%$ from all cases in the world (WHO, 2019). $P$. falciparum as the most dangerous species of Plasmodium can cause severe manifestations such as severe anemia, metabolic acidosis, multiorgan failure, cerebral malaria, and coma that is leading to death (Maier et al., 2019).

Based on the World Health Organization guidelines, malaria infection should be treated with artemisinin-based combination (ACT) therapies. Antimalarial drug resistance had been found in many cases and it has been a serious health issue for more than 5 decades. In an area with a high level of resistance, ACT as the first-line treatment for malaria. It also has a risk to be resistant (Fairhurst and Dondorp, 2016). In 2014, the molecular resistance marker against artemisinin was identified. Mutation in the receptor of the parasite was found to be related to delay clearance of the parasite in vitro and in vivo experiments (WHO, 2017).

Therefore, alternative treatment is needed to overcome antimalarial drug resistance by exploring natural resources, one of them, is lollyfish (Holothuria atra). $H$. atra has been known to have several active substances, such as chlorogenic acid, pyrogallol, rutin, coumaric acid, catechin, ascorbic acid, and artemisinin (Pangestuti and Arifin, 2018).

P. falciparum has an enzyme named Plasmodium falciparum orotidine 5'-monophosphate decarboxylase (PfOMPDC), that facilitates the pyrimidine synthesis pathway (Krungkrai and Krungkrai, 2016). Derivates of pyrimidine have an important role as the nitrogen base in deoxyribonucleic acid (DNA) and ribonucleic acid (RNA). Different from human cells, the malaria parasite can not fulfill its need for pyrimidine from 
extracellular but depends on nucleotide synthesized through the biosynthesis de novo pathway (Krungkrai and Krungkrai, 2016). In this research, $P f O M P D C$ is chosen for its important role in the malaria parasite's development process. If PfOMPDC can be inhibited, the development rate of the parasite will slow down. Therefore, PfOMPDC can be a candidate of the new antimalarial drug. Moreover, there is not much research that explains about inhibition of PFOMPDC and its reaction with antimalarial drugs.

In silico method could be used to discover the interactions among active substances with PfOMPDC up to the molecular level. In silico is a first step to find drugs candidate before in vitro or in vivo experiments (Wadood et al., 2013). The objective of this research was to prove the inbihition effect of active substances of $H$. atra against the development of $P$. falciparum.

\section{Materials and Methods}

This research was using the one-shot experimental study type with in silico as the method and was done in Laboratorium Biomolekuler \& Bioinformatika INBIO Lowokwaru, Malang, East Java, Indonesia from March until June 2020. The independent variable was the active substances in $H$. atra, the dependent variable was the development of $P$. falciparum.

\section{Protein structure and ligand}

Active substances from $\mathrm{H}$. atra was obtained from the database of PubChem (https://pubchem.ncbi. nlm.nih.gov/). The three-dimension structure of PfOMPDC is obtained through the database of Laboratorium INBIO or from Research Collaboratory for Structural Bioinformatics Protein Data Bank (RSCB PDB) (https://www.rcsb.org/) (Zardecki et al., 2016).

\section{Potential prediction of the substances}

The substances from $H$. atra were analyzed for their potential using WAY2DRUG PASS prediction (http://pharmaexpert.ru/PASSonline/index.php). The probability to be active $(\mathrm{Pa})$ was a number that described the potential of a tested substance. Pa was obtained by comparing the structure of substances with a substance that was proven as antimalarial. A substance was predicted to have a high potential as antiprotozoal (Plasmodium) if the $\mathrm{Pa}$ score was more than 0.7 and a substance was predicted to has the potential as antiprotozoal if the Pa score was between 0.3 and 0.7 (Filimonov et al., 2018).

\section{Prediction of the pathway}

The analysis of the interactions between the substances and protein target was obtained from STITCH DB Version 5.0 (http://stitch.embl.de/) with $P$. falciparum as the organism model with medium confident (0.4) until 50 predictions of interaction (Szklarczyk et al., 2016).

\section{Molecular docking}

Prediction of the interaction strength between receptor and ligand, based on their binding affinity can be obtained through docking. Stronger interaction was indicated by more negative scores. If the score of the tested substance was near to the control score, it could be predicted that the substance had an antagonist activity to the target protein. The protein target of this docking was the crystal structure of Plasmodium falciparum OMP Decarboxylase in complex with inhibitor 4-(2-hydroxy4-methoxyphenyl)-4-oxobutanoic acid (HMOA) (PDB ID 3VI2 Chain A). The result of molecular docking was visualized by PyMol 2.3.1. The interaction of amino acid was obtained through LigPlot 2.1 . Docking was specifically done by imitating the interaction of orotidine 5'-monophosphate (OMP) with a control inhibitor (Meng et al., 2012). Grid box docking was as follows:

$\begin{array}{lll}\text { 1. } & \text { receptor } & =\text { omp.pdbqt } \\ \text { 2. } & \text { exhaustiveness } & =8 \\ \text { 3. } & \text { num_modes } & =9 \\ \text { 4. } & \text { center_x } & =21.7476516009 \\ \text { 5. } & \text { center_y } & =34.5747086973 \\ \text { 6. } & \text { center_z } & =13.3276803454 \\ \text { 7. } & \text { size_x } & =15.6889492817 \\ \text { 8. } & \text { size_y } & =12.5325716884 \\ \text { 9. } & \text { size_z } & =12.4958716912 \\ \text { 10. } & \text { cpu } & =7\end{array}$

Prediction of absorption, distribution, metabolism, and excretion (ADME)

Physicochemical and pharmacokinetic of a substance that has the potential to be a drug can be found through absorption, distribution, metabolism, and excretion (ADME) analysis by using SWISS ADME (http://www.swissadme.ch/index.php) (Daina et al., 2017). To observe the result, Lipinski's rule of 5 was used. The following was Lipinski's rule of 5: Less than equal to 5 hydrogen bond donors (total bond of nitrogen-hydrogen, oxygen-hydrogen); less than equal to 10 hydrogen bond acceptors (all nitrogen atom or oxygen); molecular weight is less than 500 dalton (Benet et al., 2016).

Blood-brain barrier (BBB) analysis was used to predict whether a substance can penetrate the blood-brain barrier. Estimation of absorbed 
substance was predicted through the human intestinal absorption (HIA) score. A higher HIA score indicated a more absorbed substance.

\section{Prediction of toxicity}

The prediction of toxicity was obtained from Pro-Tox (http://tox.charite.de/protox_Il/) (Banerjee et al., 2018). The parameter in toxicity test as follows:

1. LD50 (mg. kg-1 wt) was a median of a lethal dose, which means a dose where $50 \%$ of the tested subject died because of the substance exposure. Toxicity class:

a. Class I : fatal if swallowed (LD50 $\leq 5)$

b. Class II : fatal if swallowed $(5<\operatorname{LD} 50 \leq 50)$

c. Class III : toxic if swallowed $(50<$ LD50 $\leq$ 300)

2. The toxicity level referred to the globally harmonized system (GHS) (Erhirhie et al., 2018).

\section{Result and Discussion}

\section{Active substances in $H$. atra and its potential prediction}

The active substances in $H$. atra consisted of chlorogenic acid, pyrogallol, rutin, coumaric acid, catechin, and ascorbic acid (Table 1). Pyrogallol (Pa 0.252 ) and catechin ( $\mathrm{Pa}$ 0.284) had the potential to be antiprotozoal although the $\mathrm{Pa}$ score was not higher than artemisinin ( $\mathrm{Pa}$ 0.954). The potential to be antiprotozoal for coumaric acid, ascorbic acid, and chlorogenic acid were not found. All of the substances showed in Table 1 had potential as antiparasitic.

There was no previous research on pyrogallol as antimalarial and antiparasitic, but Li and Wang (2015) showed that pyrogallol was formed from hydrolysis of tannin to gallic acid then to pyrogallol. Lutgen's research showed that tannin binded to a special protein in $P$. falciparum and caused morphological changes, physiology, and function of the host's cells which interrupted the development of the parasite. Tannin can reduce the motility of Pseudomonas aeruginosa and lowering the population of protozoa in the stomach of ruminate animals (Lutgen, 2018). Gallic acid also had an antimalarial activity for its interaction with dihydrofolate reductase (DHFR) enzyme in $P$. falciparum and inhibited that enzyme based on docking visualization with in silico (Arsianti et al., 2017). This was conformable with the result of the prediction of pyrogallol in Table 1.
Catechin from Garcinia celebica leaf can inhibit the development of $P$. falciparum in trophozoite and schizont phase through oxidative stress induction mechanism (Abdulah et al., 2017). Catechin also acted as antiparasitic and it was proven that catechin can inhibit the development of Entamoeba hystolytica and Giardia intestinalis in certain levels (Al-Jaber et al., 2010). This was conformable with the test result in Table 1 with catechin's Pa 0.284 as antimalaria and Pa 0.191 as antiparasitic even though not as high as $\mathrm{Pa}$ of artemisinin.

Overall prediction result of substance potential from $H$. atra stated that $H$. atra had an activity as antimalarial and antiparasitic for Plasmodium specifically although the $\mathrm{Pa}$ score was not as high as artemisinin.

\section{Prediction of active substances pathway against PFOMPDC}

Visualization by STITCH DB of the interaction between substances and PfOMPDC was presented in Figure 1 and 2. There was no direct interaction between active substances and PfOMPDC, but there were indirect interactions between pyrogallol, catechin, and ascorbic acid with PfOMPDC through $\mathrm{CO}_{2}$. Chlorogenic acid should become catechin first before having an indirect interaction with PfOMPDC. There were indirect and direct interactions between OMP with PfOMPDC. The OMP and PfOMPDC interacted indirectly through $\mathrm{CO}_{2}$. Artemisinin and coumaric acid did not have direct or indirect interaction with PfOMPDC.

Biosynthesis de novo of pyrimidine nucleotide needed an amide group from glutamine, carbon, and the amino acid group from aspartate. A group of $\alpha$ carboxyl carbon from aspartate will be released as $\mathrm{CO}_{2}$ (Stipanuk and Caudill, 2012). The release of $\mathrm{CO}_{2}$ will stabilize decarboxylation which catalyzed by OMPDC and reused for the next biosynthesis de novo of pyrimidine nucleotide (Richard et al., 2018).

There are no previous research on interaction and reaction between pyrogallol, chlorogenic acid, catechin, and coumaric acid with PfOMPDC. Pyrogallol, chlorogenic acid, catechin, and coumaric acid were included in the phenol group (Lima et al., 2016). The increasing rate of $\mathrm{CO}_{2}$ will accelerate the reduction of $\mathrm{Fe}^{3+}$ through phenolic compounds which was very crucial to fulfilling the cell needs of $\mathrm{Fe}^{2+}$. The phenolic compound can be advantageous for human healthy cells and can be an inhibitor for the development of the parasite inside human cells because both PFOMPDC and phenolic compounds need $\mathrm{CO}_{2}$ to work on their pathway. 
ILMU KELAUTAN: Indonesian Journal of Marine Sciences December 2020 Vol 25(4):135-142

Table 1. Substance potential as antiprotozoal and antiparasitic using WAY2DRUG PASS

\begin{tabular}{ccc}
\hline Substance & Antiprotozoal (Plasmodium) $(\mathrm{Pa})$ & Antiparasitic $(\mathrm{Pa})$ \\
\hline Coumaric acid & Not found & 0.466 \\
Artemisinin & 0.954 & 0.857 \\
Pyrogallol & 0.252 & 0.329 \\
Catechin & 0.284 & 0.191 \\
Ascorbic Acid & Not found & 0.306 \\
Chlorogenic Acid & Not found & 0.410 \\
\hline
\end{tabular}

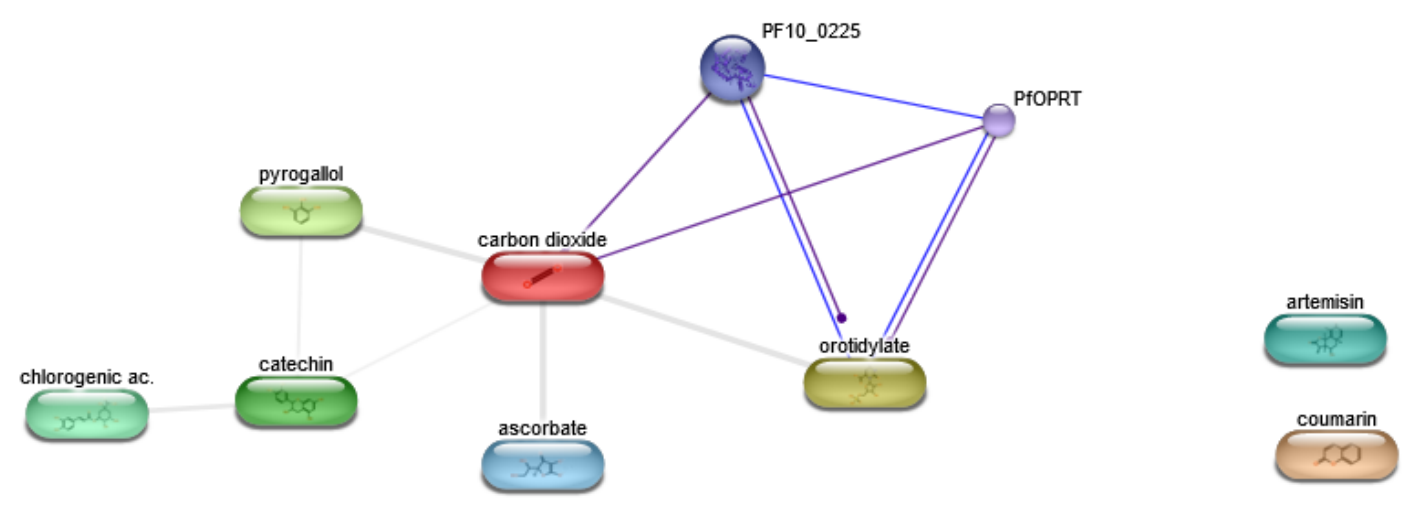

Figure 1. Pathway prediction of active substances in Holothuria atra against PfOMPDC

Table 2. Information for pathway prediction of active substances in Holothuria atra against PfOMPDC

\begin{tabular}{|c|c|}
\hline Input & Information \\
\hline Carbon dioxide & $\begin{array}{l}\text { A colorless, odorless gas that can be formed by the body and is necessary for the respiration cycle of } \\
\text { plants and animals. }(44.0 \text { g.mol-1) }\end{array}$ \\
\hline Coumarin & $\begin{array}{l}\text { Coumarin }(2 \mathrm{H} \text {-chromen-2-one) is a fragrant organic chemical compound in the benzopyrone chemical } \\
\text { class, which is a colorless crystalline substance in its standard state. It is a natural substance found in } \\
\text { many plants. (146.1 g.mol-1) }\end{array}$ \\
\hline Orotidylate & $\begin{array}{l}\text { Orotidine } 5^{\prime} \text {-monophosphate (OMP), also known as orotidylic acid, is a pyrimidine nucleotide which is } \\
\text { the last intermediate in the biosynthesis of uridine monophosphate. OMP is formed from orotate and } \\
\text { phosphoribosyl pyrophosphate by the enzyme Orotate phosphoribosyl transferase. }(368.2 \text { g.mol-1) }\end{array}$ \\
\hline Pyrogallol & A trihydroxybenzene or dihydroxy phenol that can be prepared by heating gallic acid. $(126.1$ g.mol-1) \\
\hline Catechin & $\begin{array}{l}\text { Catechin is a flavan-3-ol, a type of natural phenol and antioxidant. It is a plant secondary metabolite. It } \\
\text { belongs to the group of flavan-3-ols (or simply flavanols), part of the chemical family of flavonoids. } \\
\qquad(290.3 \text { g.mol-1) }\end{array}$ \\
\hline Chlorogenic ac. & Chlorogenic acid (354.3 g.mol-1) \\
\hline Artemisin & Artemisia (262.3 g.mol-1) \\
\hline Ascorbate & $\begin{array}{l}\text { A six-carbon compound related to glucose. It is found naturally in citrus fruits and many vegetables. } \\
\text { Ascorbic acid is an essential nutrient in human diets, and necessary to maintain connective tissue and } \\
\text { bone. Its biologically active form, vitamin C, functions as a reducing agent and coenzyme in several } \\
\left.\text { metabolic pathways. Vitamin C is considered an antioxidant. [PubChem] ( } 214.2 \text { g.mol- }^{-1}\right)\end{array}$ \\
\hline PF10_0225 & Orotidine 5'-phosphate decarboxylase (323 aa) \\
\hline PfOPRT & Orotate phosphoribosyl transferase (281aa) \\
\hline
\end{tabular}

Current Organism: Plasmodium falciparum

NCBI taxonomy ld: 5833

Other names: Laverania, P. (Laverania), P. falciparum, Plasmodium (Laverania), Plasmodium (Laverania) falciparum, Plasmodium falciparum, malaria parasite $P$. falciparum 
Ascorbic acid has an interaction with PfOMPDC indirectly through $\mathrm{CO}_{2}$. Increasing $\mathrm{CO}_{2}$ will speed up the biosynthesis of ascorbic acid and pathway of gen regeneration (Muthusamy et al., 2019). Through this mechanism, ascorbic acid and PfOMPDC will compete for their needs of $\mathrm{CO}_{2}$.

The molecular docking was done to know the binding affinity for each substance with PfOMPDC. The more negative binding affinity score indicated stronger interaction. The target of this molecular docking was the crystal structure complex of PfOMPDC with HMOA as the control inhibitor. In Table 3, chlorogenic acid had the strongest affinity (most negative score) to PfOMPDC, followed by catechin, artemisinin, and control. Coumaric acid and pyrogallol had the weakest affinity towards PfOMPDC.

Based on Table 4, catechin had three hydrophobic bonds and three hydrogen bonds in amino acid residue that was the same with control. If the result from LigPlot (Table 4) merged with binding affinity score (Table 3 ), the substance that had the best interaction with PfOMPDC is catechin.

There was no interaction between artemisinin and coumaric acid with PFOMPDC in STITCH DB visualization but there was in molecular docking that can be seen in Figure 3 and Figure 4, it is because STITCH DB is only for prediction and the result is also based on previous researches (Szklarczyk et al., 2016).

\section{Prediction of Absorption, Distribution, Metabolism, and Excretion (ADME)}

ADME of each substance was showed in Table 5, each substance in the table met the criteria of Lipinski. Catechin was the safest substance in $H$. atra because of its high score of water solubility, Gastro-Intestinal (GI) absorption, and bioavailability. Coumaric acid was water-soluble, its Gl absorption and bioavailability score are high too. Pyrogallol and ascorbic acid are very water-soluble, its GI absorption and bioavailability score are high. Chlorogenic acid is very water-soluble but its Gl absorption and bioavailability score are low, it makes chlorogenic acid is less suitable to be used as a drug. Coumaric acid and pyrogallol are permeable to BBB, therefore, the two substances have the potential to cure cerebral malaria (Nishanth and Schlüter, 2019).

\section{Prediction of toxicity}

The more potent or toxic the substance, lower the LD50 and smaller the dose needed to cause death. The best substance in $H$. atra to inhibit PfOMPDC was catechin with LD50 10.000 mg. kg-1 wt and the toxicity class is 6 (the safest class). Catechin is safer than artemisinin with LD50 4.228 mg. $\mathrm{kg}^{-1}$ wt and toxicity class 5 . The following is chlorogenic acid with LD50 5.000 mg. kg-1 wt with toxicity class 5 , next is ascorbic acid with LD50 3.367 mg. $\mathrm{kg}^{-1}$ wt and toxicity class 5 . Coumaric acid has LD50 2.850 mg.kg-1 wt with toxicity class 5 and

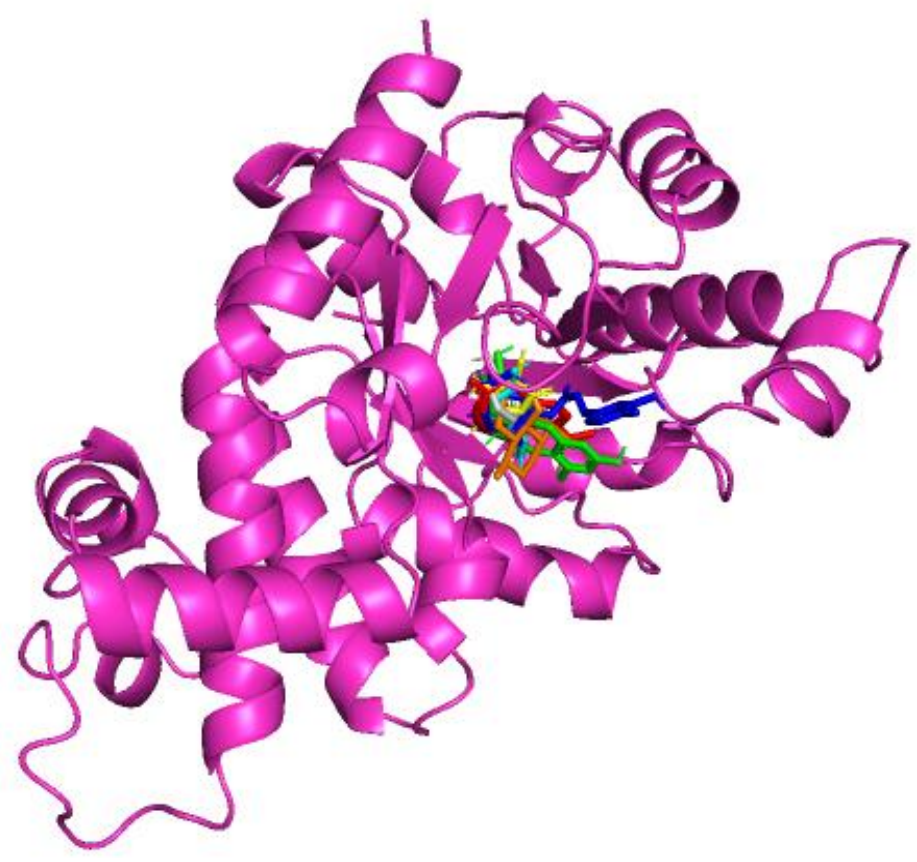

Figure 3. Visualization from docking result of OMP-ligand (color indicator is in Table 3) 
ILMU KELAUTAN: Indonesian Journal of Marine Sciences December 2020 Vol 25(4):135-142

Table 3. Molecular docking result between PfOMPDC and ligand

\begin{tabular}{ccc}
\hline Substance & Binding affinity score(kcal.mol-1) & Color visualization \\
Coumaric acid & -5.3 & Cyan \\
Artemisinin & -7.3 & Orange \\
Pyrogallol & -5.3 & Grey \\
Catechin & -7.4 & Green \\
Ascorbic acid & -5.9 & Yellow \\
Chlorogenic acid & -7.6 & Blue \\
Kontrol & -6.2 & Red \\
\hline
\end{tabular}

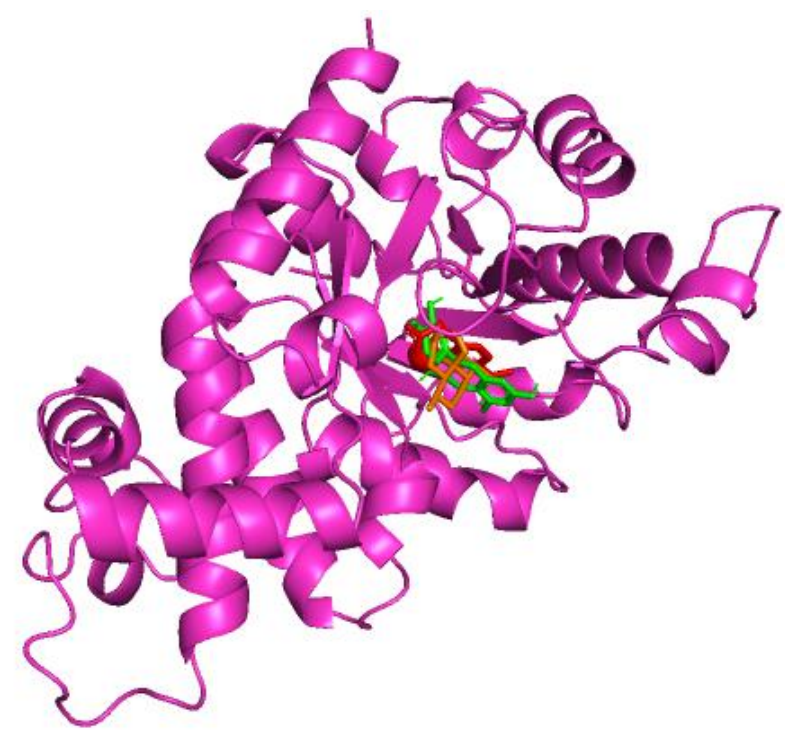

Figure 4. Visualization from best docking result of OMP-ligand (color indicator is in Table 3)

Table 4. Visualization of OMP LigPlot docking

\begin{tabular}{ccc}
\hline Substance & Hydrophobic bond & Hydrogen bond \\
\hline Control & THR195 PRO264 GLY293 ILE266 GLN269 & LYS138 LYS102 ASN291 GLY265 ALA268 \\
Catechin & THR195 GLY265 ARG294 ILE292 GLY293 & LYS138 ASN291 ILE206 ASP23 LYS102 ASN104 \\
\hline
\end{tabular}

Information: bold datas above indicate the same amino acid residue from control and ligand

Table 5. ADME result by SWISS ADME

\begin{tabular}{|c|c|c|c|c|c|c|}
\hline & Coumaric acid & Pyrogallol & Artemisinin & Catechin & Ascorbic acid & Chlorogenic acid \\
\hline Molecular weight & 164.16 g. $\mathrm{mol}^{-1}$ & 126.11 g.mol-1 $^{-1}$ & 282.33 g.mol-1 & 290.27 g.mol-1 & 176.12 g.mol-1 & 354.31 g.mol$^{-1}$ \\
\hline $\begin{array}{l}\text { Hydrogen Bond } \\
\text { Acceptors }\end{array}$ & 3 & 3 & 5 & 6 & 6 & 9 \\
\hline $\begin{array}{l}\text { Hydrogen Bond } \\
\text { Donors }\end{array}$ & 0 & 0 & 0 & 0 & 0 & 0 \\
\hline Atoms & 12 & 9 & 20 & 21 & 12 & 25 \\
\hline Water solubility & Soluble & Very soluble & Soluble & Soluble & Very soluble & Very soluble \\
\hline Gl Absorption & High & High & High & High & High & Low \\
\hline BBB permeant & + & + & + & - & - & - \\
\hline Bioavailability & 0.56 & 0.55 & 0.55 & 0.55 & 0.56 & 0.11 \\
\hline
\end{tabular}

Lipinski's rule of 5 :

1. Less than equal to 5 hydrogen bond donors (total bond of nitrogen-hydrogen, oxygen-hydrogen)

2. Less than equal to 10 hydrogen bond acceptors (all nitrogen atom or oxygen)

3. Molecular weight is less than 500 dalton. 
Table 6. Toxicity prediction by Pro-Tox

\begin{tabular}{|c|c|c|c|c|c|c|}
\hline & Coumaric acid & Pyrogallol & Artemisinin & Catechin & Ascorbic acid & Chlorogenic acid \\
\hline $\begin{array}{l}\text { Prediction of } \\
\text { LD50 (mg.kg-1 } \\
\text { wt) }\end{array}$ & 2.850 & 300 & 4.228 & 10.000 & 3.367 & 5.000 \\
\hline $\begin{array}{l}\text { Prediction of } \\
\text { toxicity (class) }\end{array}$ & 5 & 3 & 5 & 6 & 5 & 5 \\
\hline
\end{tabular}

pyrogallol has very low LD50 that is $300 \mathrm{mg}^{\mathrm{kg}} \mathrm{kgt}^{-1} \mathrm{wt}$ and the toxicity class is 3 . This makes pyrogallol become the most toxic substance from all other substances in H. atra (Raj et al., 2013).

\section{Conclusion}

There were two substances in $H$. atra i.e. pyrogallol and catechin that had the potential as antimalarial. Pathway prediction of the substances stated that there was indirect inhibition between pyrogallol, chlorogenic acid, catechin, and ascorbic acid with PfOMPDC through $\mathrm{CO}_{2}$. The binding affinity score of catechin and chlorogenic acid were higher than other substance even artemisinin too but catechin was the only substance that had similarity in amino acid residue with the control (as seen in Table 4). Catechin was the safest substance based on ADME and toxicity prediction. Therefore, active substances in lollyfish $(H$. atra) had inhibitory effects against the development of $P$. falciparum based on in silico study.

\section{Acknowledgement}

High appreciation is given to Faculty of Medicine Hang Tuah University and Laboratorium Biomolekuler \& Bioinformatika INBIO Indonesia for the contribution in establishing research design, acquisition of data in silico method, supplying documents/photographs or analysis and interpretation of data.

\section{References}

Abdulah, R., Suradji, E.W., Subarnas, A., Supratman, U., Sugijanto, M., Diantini, A., Lestari, K., Barliana, M.I., Kawazu, S. \& Koyama, H. 2017. Catechin isolated from Garcinia celebica leaves inhibit Plasmodium falciparum growth through the induction of oxidative stress, Pharmacogn. Mag., 13(2):301-305. doi: 10.4103/pm.pm_ 571_16.

Al-Jaber, H.I., Mosleh, I.M., Mallouh, A., Abu Salim, O.M. \& Abu Zarga, M.H. 2010. Chemical constituents of Osyris alba and their antiparasitic activities, J. Asian Nat. Prod. Res.,
12(9):814-820. doi: 10.1080/10286020.201 0.502892

Arsianti, A., Astuty, H., Fadilah, Bahtiar, A., Tanimoto, H. \& Kakiuchi, K. 2017. Design and screening of gallic acid derivatives as inhibitors of malarial dihydrofolate reductase by in silico docking, Asian J. Pharm. Clin. Res., 10(2):330334. doi: 10.22159/ajpcr.2017.v10i2.15712.

Banerjee, P., Eckert, A.O., Schrey, A.K. \& Preissner, R. 2018. ProTox-II: A webserver for the prediction of toxicity of chemicals, Nucleic Acids Res., 46(W1):W257-W263. doi: 10.1093/nar/ gky318.

Benet, L.Z., Hosey, C.M., Ursu, O. \& Oprea, T.I. 2016. BDDCS, the Rule of 5 and drugability, Adv. Drug Deliv. Rev., 101:89-98. doi: 10.1016/j.addr. 2016.05.007.

Daina, A., Michielin, O. \& Zoete, V. 2017. SwissADME: A free web tool to evaluate pharmacokinetics, drug-likeness and medicinal chemistry friendliness of small molecules, Sci. Rep., 7(1):42717. doi: 10.1038/srep42717.

Erhirhie, E.O., Ihekwereme, C.P. \& Ilodigwe, E.E. 2018. Advances in acute toxicity testing: Strengths, weaknesses and regulatory acceptance, Interdiscip. Toxicol. 11(1):5-12. doi: 10.2478/intox-2018-0001.

Fairhurst, R.M. \& Dondorp, A.M. 2016. ArtemisininResistant Plasmodium falciparum Malaria, Microbiol. Spectr., 4(3): 455-467. doi: 10.1128/microbiolspec.ei10-0013-2016.

Filimonov, D.A., Druzhilovskiy, D.S., Lagunin, A.A., Gloriozova, T.A., Rudik, A.V., Dmitriev, A.V., Pogodin, P.V. \& Poroikov, V.V. 2018. Computeraided prediction of biological activity spectra for chemical compounds: opportunities and limitation', Biomed. Chemi. Res. Methods, 1(1):1-21. doi: 10.18097/bmcrm00004.

Krungkrai, S.R. \& Krungkrai, J. 2016. Insights into the pyrimidine biosynthetic pathway of human malaria parasite Plasmodium falciparum as 
chemotherapeutic target, Asian Pacific J. Trop. Med., 9(6):525-534. doi: 10.1016/j.apjtm. 2016.04.012.

Li, W. \& Wang, C. 2015. Biodegradation of gallic acid to prepare pyrogallol by Enterobacter aerogenes through substrate induction, BioResources, 10(2):3027-3044. doi: 10.153 76/biores.10.2.3027-3044.

Lima, V.N., Oliveira-Tintino, C.D.M., Santos, E.S., Morais, L.P., Tintino, S.R., Freitas, T.S., Geraldo, Y.S., Pereira, R.L. S., Cruz, R.P., Menezes, I.R.A. \& Coutinho, H.D.M. 2016. Antimicrobial and enhancement of the antibiotic activity by phenolic compounds: Gallic acid, caffeic acid and pyrogallol, Microb. Pathog., 99:56-61. doi: 10.1016/j.micpath.2016.08.004.

Lutgen, P. 2018. Tannins in Artemisia: the hidden treasure of prophylaxis, Pharm. Pharmacol. Int. J., 6(3):176-181. doi: 10.15406/ppij.2018. 06.00173.

Maier, A.G., Matuschewski, K., Zhang, M. \& Rug, M. 2019. Plasmodium falciparum, Trends Parasitol. 35(6):481-482. doi: 10.1016/j.pt. 2018.11.010.

Meng, X.Y., Zhang, H.X., Mezei, M. \& Cui, M. 2012. Molecular Docking: A Powerful Approach for Structure-Based Drug Discovery, Curr. Comput. Aided Drug Des., 7(2):146-157. doi: 10.217 4/157340911795677602.

Muthusamy, M., Hwang, J.E., Kim, S.H., Kim, J.A., Jeong, M.J., Park, H.C. \& Lee, S.I. 2019. Elevated carbon dioxide significantly improves ascorbic acid content, antioxidative properties and restricted biomass production in cruciferous vegetable seedlings, Plant Biotechnol. Rep., 13(3):293-304. doi: 10.100 7/s11816-019-00542-3.

Nishanth, G. \& Schlüter, D. 2019. Blood-Brain Barrier in Cerebral Malaria: Pathogenesis and Therapeutic Intervention, Trends Parasitol., 35(7):516-528. doi: 10.1016/j.pt.2019.04.010.

Pangestuti, R. \& Arifin, Z. 2018. Medicinal and health benefit effects of functional sea cucumbers, J. Tradit Complement. Med.,
8(3):341-351. doi: 10.1016/j.jtcme.2017.06. 007.

Putra, T.R.I. 2011. Malaria dan Permasalahannya, J. Kedok. Syiah Kuala, 11(2):103-114.

Raj, J., Chandra, M., Dogra, T.D., Pahuja, M. \& Raina, A. 2013. Determination of median lethal dose of combination of endosulfan and cypermethrin in wistar rat, Toxicol. Int., 20(1):1-5. doi: 10.4103/0971-6580.111531.

Richard, J.P., Amyes, T.L. \& Reyes, A.C. 2018. Orotidine 5'-Monophosphate Decarboxylase: Probing the Limits of the Possible for Enzyme Catalysis, Acc. Chem. Res., 51(4):960-969. doi: 10.1021/acs.accounts.8b00059.

Stipanuk, M. \& Caudill, M. 2012. Biochemical, Physiological, and Molecular Aspects of Human Nutrition 3rd Edition, Med. Sci. Sports Exerc. ELSEVIER, p. 448.

Szklarczyk, D., Santos, A., Von Mering, C., Jensen, L.J., Bork, P. \& Kuhn, M. 2016. STITCH 5: Augmenting protein-chemical interaction networks with tissue and affinity data. Nucleic Acids Res., 44(D1):380-384. doi: 10.1093/nar /gkv1277.

Wadood, A., Ahmed, N., Shah, L., Ahmad, A., Hassan, H. \& Shams, S. 2013. In-silico drug design: An approach which revolutionarised the drug discovery process, Drug Design and Deliv., 1(1):1-4. doi: 10.13172/2054-4057-1-1-1119.

WHO (2019) World Malaria Report 2019, World Health Organization. Available at: https://www.who.int/publications-detailredirect/world-malaria-report-2019. Acessed 3 March 2020

World Health Organization (2017) 'Artemisinin and artemisinin-based combination therapy resistance', WHO.

Zardecki, C., Dutta, S., Goodsell, D.S., Voigt, M. \& Burley, S.K. 2016. RCSB Protein Data Bank: A Resource for Chemical, Biochemical, and Structural Explorations of Large and Small Biomolecules, J. Chem. Edu., 93(3):569-575. doi: 10.1021/acs.jchemed.5b00404. 